\title{
New Developments in Dual-Labeled Molecular Imaging Agents
}

\author{
Servando Hernandez Vargas, Sukhen C. Ghosh, and Ali Azhdarinia \\ Brown Foundation Institute of Molecular Medicine, McGovern Medical School, The University of Texas Health Science Center at \\ Houston, Houston, Texas
}

\begin{abstract}
Intraoperative detection of tumors has had a profound impact on how cancer surgery is performed and addresses critical unmet needs in surgical oncology. Tumor deposits, margins, and residual cancer can be imaged through the use of fluorescent contrast agents during surgical procedures to complement visual and tactile guidance. The combination of fluorescent and nuclear contrast into a multimodality agent builds on these capabilities by adding quantitative, noninvasive nuclear imaging capabilities to intraoperative imaging. This review focuses on new strategies for the development and evaluation of targeted dual-labeled molecular imaging agents while highlighting the successful first-inhuman application of this technique.
\end{abstract}

Key Words: fluorescence-guided surgery; dual-labeling; multimodality imaging; cancer surgery

J Nucl Med 2019; 60:459-465

DOI: 10.2967/jnumed.118.213488

\section{$\mathbf{S}$}

urgery is widely considered the gold standard for treatment of solid tumors. To increase the precision of surgical oncology, intraoperative imaging has been used to overcome difficulties in identifying small lesions, surgical margins, and cancer-positive lymph nodes $(1,2)$. In particular, the intraoperative application of fluorescent contrast agents has shown significant promise in color coding the surgical field of view and enabling surgeons to more effectively distinguish tumor from adjacent nontumor tissues. Fluorescenceguided surgery (FGS) is based on the use of fluorescent dyes that accumulate in tumors either passively or through active processes such as receptor-mediated uptake, by conjugation to a targeting moiety. The number of FGS agents under clinical investigation is a testament to the growing importance of the field and its high translational significance. Since the initial first-in-human study by Van Dam et al. in 2011 (3), more than 1,000 procedures and agents that use fluorescence-based surgical guidance have been listed in clinicaltrials.gov (4). This growth is due in large part to several key advantages of FGS over existing intraoperative imaging

\footnotetext{
Received Nov. 21, 2018; revision accepted Jan. 24, 2019.

For correspondence or reprints contact: Ali Azhdarinia, The University of Texas Health Science Center at Houston, 1881 East Rd., 3SCR6.4680, Houston, TX 77054.

E-mail: ali.azhdarinia@uth.tmc.edu

Published online Feb. 7, 2019.

COPYRIGHT @ 2019 by the Society of Nuclear Medicine and Molecular Imaging
}

tools. Unlike radioguided surgery, which uses audible signals to detect sites of radiotracer accumulation, FGS produces real-time images that provide a spatial map of the surgical field of view and enhance the ability of surgeons to detect tumors, identify margins, and assess residual tumor tissue in the wound bed. In addition, FGS with targeted agents gives functional readouts that complement the anatomic information obtained from intraoperative ultrasound. Moreover, researchers have access to a multitude of commercially available fluorescent dyes that, first, emit in a particular region of the electromagnetic spectrum and can be hand-picked for specific clinical applications and, second, possess different reactive groups that enable straightforward bioconjugation to targeting agents (Fig. 1).

Despite the clear utility of FGS, the low-energy photons emitted by fluorescent contrast agents are highly scattered in tissues and limit the effectiveness of this approach for detecting lesions that reside deep in tissues. This inherent limitation of biomedical optics also leads to an inability to quantitatively measure the uptake of a fluorescent agent in tissues and obscures our understanding of its in vivo biodistribution. However, since the detection sensitivity of optical imaging is similar to PET and SPECT (picomolarfemtomolar range), fluorescent and radioactive labels have been effectively combined into a dual labeling strategy that produces a multimodality (or hybrid) imaging agent that overcomes these limitations. Aspects of agent design, such as the selection of dyes, chelators, and radionuclides, have been reviewed elsewhere in detail (5-7). The multimodality imaging approach is highly complementary and merges the quantitative capability of nuclear-based imaging methods with the high spatial resolution of optical imaging. Dual labeling also allows clinicians to perform preoperative nuclear imaging for treatment planning and intraoperative fluorescence imaging for tumor resection with the same agent. Importantly, dual labeling ensures that signals obtained from each modality are collected from the same agent, thus enabling cross-validation of each modality. In a proof-of-concept study, van der Poel et al. showed the benefits of using a nontargeted multimodality imaging agent for improved sentinel lymph node detection in prostate cancer with a self-assembling construct based on a nanocolloid and the near-infrared fluorescent (NIRF) dye indocyanine green (excitation/emission wavelengths [Ex/Em], 


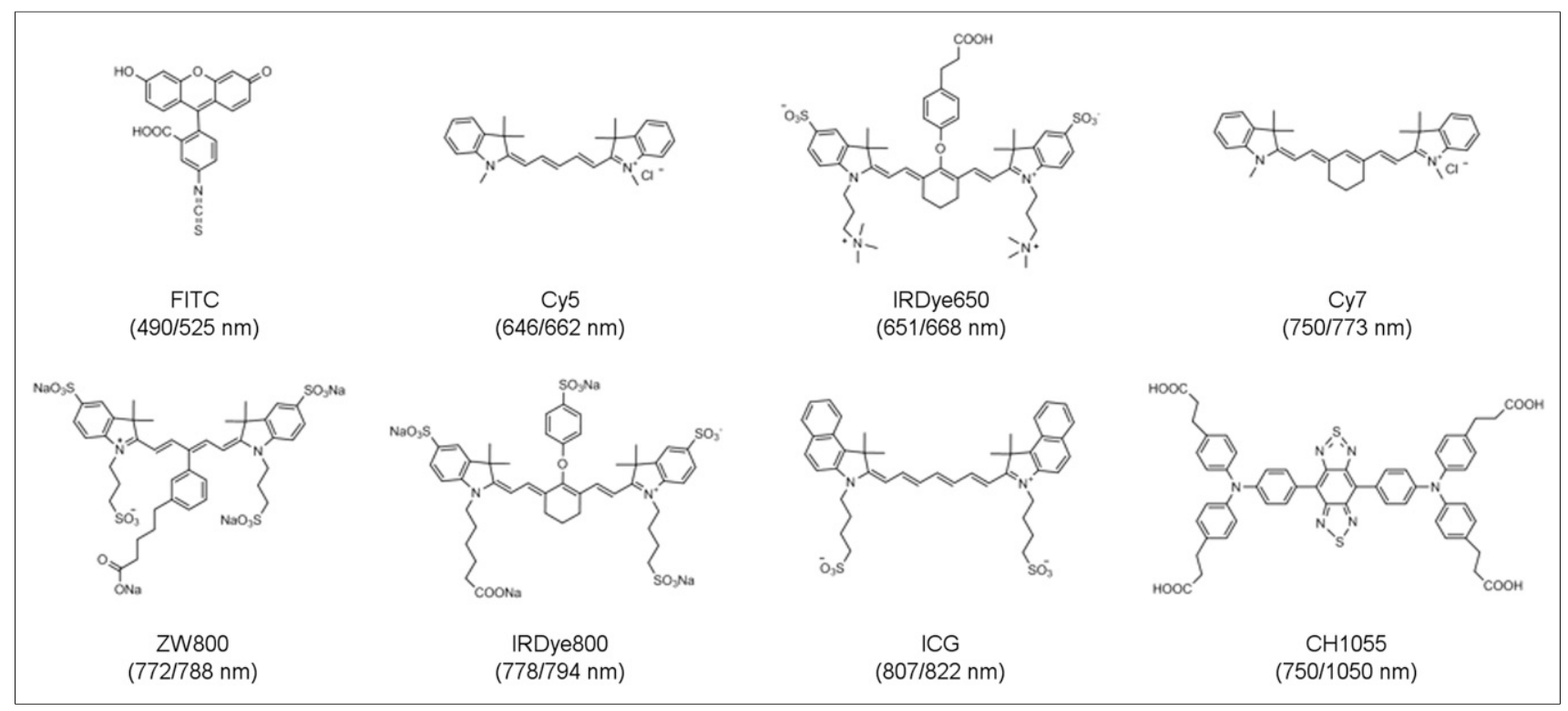

FIGURE 1. Chemical structures of dyes commonly used in the synthesis of dual-labeled imaging agents. (Absorption and emission spectra are shown as Ex/Em.)

$807 / 822 \mathrm{~nm})(8)$. Imaging with the NIRF/nanocolloid has since been expanded to a heterogeneous patient population, and results from a prospective clinical study suggest that sentinel lymph node biopsies performed with multimodality imaging are more accurate than visual blue dye-based detection alone (9). Similar clinical trials for targeted duallabeled agents are envisioned, and the robust preclinical pipeline of agents based on antibodies and peptides has demonstrated excellent translational potential.

\section{ANTIBODY-BASED AGENTS}

Monoclonal antibodies (mAbs) are widely used in the fields of imaging, therapy, and drug delivery because of their excellent specificity for target antigens and low uptake in nontarget tissues. The high molecular weight of mAbs allows conjugation of multiple dye and chelator complexes without significant loss of immunoreactivity, making these molecules excellent candidates for dual labeling. Several strategies have been used to dual-label mAbs, with the most straightforward approach involving the formation of amide bonds between the side chains of lysine residues within the protein and activated ester analogs of dyes and chelators. This method was used by Hekman et al. to dual-label the anticarcinoembryonic antigen (CEA) mAb labetuzumab with ${ }^{111}$ In (via diethylenetriaminepentaacetic acid [DTPA] conjugation) and IRDye800 (Ex/Em, 778/794 nm) for imageguided resection of CEA-overexpressing intraperitoneal tumors in a model (10). Since many tumors are grossly identifiable during surgery, the efficacy of dual-labeled agents is related to their ability to detect very small tumors that could be missed by the naked eye. Accordingly, the authors used a pulmonary micrometastasis mouse model and showed the feasibility of detecting microlesions $(<2 \mathrm{~mm})$ using
${ }^{111}$ In-DTPA-labetuzumab-IRDye800 for noninvasive SPECT imaging, followed by fluorescence-guided resection. Remarkably, submillimeter tumor colonies that were not identifiable on macroscopic inspection could be identified using fluorescence imaging, for higher rates of resection. Odenthal et al. used a similar dual labeling approach for detecting invasive human head and neck squamous cell carcinoma in xenografts (11). In their work, a humanized anti-CD44v6 mAb, bivatuzumab, was conjugated to IRDye 800 and ${ }^{111}$ In to yield ${ }^{111}$ In-DTPA-bivatuzumab-IRDye800. The dual-labeled mAb was able to noninvasively detect tumors using nuclear and optical imaging with high agent accumulation $(54 \pm 11$ percentage injected dose per gram $[\% \mathrm{ID} / \mathrm{g}])$. Notably, detection of bivatuzumab-IRDye800 with a clinical fluorescence imaging system (QMI Spectrum; Quest Medical Imaging) allowed the image-guided resection of submillimeter-sized residues $(0.7-2 \mathrm{~mm})$ containing only a few thousand cells, as determined by NIRF scanning and high-resolution immunohistochemistry analysis.

Bispecific antibody fragments have been used to target 2 different antigens and increase overall tumor uptake (12). Luo et al. applied this concept toward the development of a dual-labeled heterobifunctional immunoconjugate for pancreatic cancer (13). Using biorthogonal click-chemistry, tetrazine functionalized CD105 and transcyclooctene (TCO)-functionalized tissue factor $\mathrm{Fab}^{\prime}$ antibody fragments were conjugated together, followed by dual labeling with ${ }^{64} \mathrm{Cu}$ and the zwitterionic NIRF dye ZW800 (Ex/Em, 772/788 nm) (14). PET/CT imaging and ex vivo pharmacokinetic studies revealed improved tumor targeting using ${ }^{64} \mathrm{Cu}$-NOTA-heterodimerZW800, with reduced liver retention when compared with either Fab fragment homodimer. NIRF imaging of resected tissues was in agreement with nuclear results, and the authors concluded that bispecific targeting of well-established 
biomarkers in FGS could potentially amplify the fluorescent signal during surgery to improve detection.

The conjugation of dyes and chelators to lysine residues is random and could occur in the binding region of mAbs and reduce bioactivity. Various bioconjugation techniques have been developed to overcome this problem, including site-specific conjugation to the $\mathrm{Fc}$ region of mAbs $(15,16)$. Houghton et al. reported the development of a site-specific dual-labeled humanized mAb (5B1) for cancer antigen 19-9 targeting in pancreatic ductal adenocarcinoma (17). The combination of glycan engineering and bioorthogonal clickchemistry permitted the synthesis of the site-specific duallabeled immunoconjugate ${ }^{89} \mathrm{Zr}^{\text {ss }}$ Dual-5B1 (NIRF dye) (Fig. 2A), which minimized concerns over reproducibility of synthesis and loss of immunoreactivity. A more than $20 \%$ increase in immunoreactivity was found in ${ }^{89} \mathrm{Zr}^{-}{ }^{-5}$ Dual-5B1 (>90\%), compared with nonspecifically modified ${ }^{89} \mathrm{Zr}-$ DFO-5B1 $(72.4 \% \pm 1.1 \%)$. Although tumor uptake for both agents was comparable at $120 \mathrm{~h}$ after agent administration $\left({ }^{89} \mathrm{Zr}^{\mathrm{ss}}\right.$ Dual-5B1, $102.9 \pm 26.0 \% \mathrm{ID} / \mathrm{g} ;{ }^{89} \mathrm{Zr}-\mathrm{DFO}-5 \mathrm{~B} 1$, $114.1 \pm 23.1 \% \mathrm{ID} / \mathrm{g}$ ) (Fig. 2B), accumulation in all nontarget tissues was lower for ${ }^{89} \mathrm{Zr}-{ }^{\text {ss }}$ Dual-5B1, with the exception of the liver ( $3 \% \mathrm{ID} / \mathrm{g}$ higher). NIRF imaging was in agreement with PET/CT results and was able to cross-validate signals at the primary tumor site, sentinel lymph nodes and primary metastases (Fig. 2C). Notably, the use of the NIRF signal allowed the detection of micrometastases that had not been previously identified during PET/CT imaging and were not apparent during macroscopic inspection. This work demonstrated how the combination of click chemistry and glycan engineering resulted in a dual-labeled 5B1 agent with enhanced in vivo properties.

To develop a universal dual labeling reagent that can be used with mAbs, Lu et al. combined ${ }^{124} \mathrm{I}$ and the clinically approved visible dye fluorescein $(\mathrm{Ex} / \mathrm{Em}, 494 / 512 \mathrm{~nm})$ to produce ${ }^{124} \mathrm{I}$-green (18). The dual-modality reagent contained a succinimide group for conjugation to the CEA-specific murine antibody $\mathrm{A} 5 \mathrm{~B} 7$, and the resulting product was administered to a CEA-expressing SW1222 xenograft mouse model. Noninvasive PET/CT imaging clearly identified tumors at 24, 48 , and $72 \mathrm{~h}$, whereas ex vivo biodistribution studies determined that the ratios between tumor and background organs were optimal at $72 \mathrm{~h}$. A whole-body fluorescence imaging system was used for intraoperative imaging, and tumors were well visualized both in vivo and ex vivo, with clear boundaries from surrounding tissues. The findings demonstrated that the dual labeling reagent could be applied in a 1-step conjugation to produce a mAb that retained binding affinity in vitro and in vivo, thus showing its feasibility for use as a generic tool for synthesis of other dual-labeled mAbs.

\section{PEPTIDE-BASED AGENTS}

Peptides are attractive models for multimodal agent development because of ease of manufacturing and scaleup via solid-phase peptide synthesis, the ability to undergo site-specific modifications to form desired bioconjugates, high chemical and in vivo stability, and favorable pharmacokinetics that enable high-contrast imaging at early time points. Moreover, dual-labeled peptides have benefited from the iterative optimization of clinically used radiotracers, allowing chemists to focus on methods to add fluorescent dyes to well-characterized radiopeptide complexes. This was shown by Zhang et al. as they developed the first dual-labeled agent for gastrin-releasing peptide receptor targeting in prostate cancer (19). Building on the initial success of the radioconjugate containing a synthetic bombesin receptor antagonist, ${ }^{68} \mathrm{Ga}-\mathrm{DOTA}-\mathrm{RM} 2$ (BAY 867548), the authors combined nuclear and NIRF (IRDye 650; Ex/Em, 651/668 nm) contrast by using the 2 amine groups of a lysine residue for conjugation to produce HZ220. Since dye conjugation could produce steric effects that negatively affect receptor binding, in vitro characterization of HZ220 was compared with the nonfluorescent precursor HZ219 and revealed a decrease in affinity after dye conjugation (half-maximal inhibitory concentration [ $\left.\mathrm{IC}_{50}\right], 21.4 \pm 7.4 \mathrm{nM}$ vs. $0.69 \pm 0.18 \mathrm{nM}$, respectively). Nonetheless, using PET/NIRF imaging and cross-validation via biodistribution studies, the authors showed in vivo receptor-targeting bioactivity and high multimodal image contrast $1 \mathrm{~h}$ after agent administration. In vivo blocking studies showed an approximately $76 \%$ reduction in tumor uptake and indicated that agent uptake was receptor-mediated, demonstrating the potential of the agent for noninvasive PET and intraoperative imaging of gastrin-releasing peptide receptor-expressing malignancies.

To develop a dye conjugation method that could be broadly applied for dual labeling, Summer et al. used the siderophorebased chelator fusarinine C (FSC) as a scaffold and conducted a proof-of-principle study (20). The authors synthesized and evaluated 2 different FSC-based agents for specific targeting of the cholecystokinin- 2 receptor and integrin $\alpha_{v} \beta_{3}$ overexpression with a minigastrin analog (MG11) or integrin $\alpha_{\mathrm{v}} \beta_{3}$ cyclic pentapeptide (RGD). Interestingly, FSC has 3 primary amines for site-specific conjugation, which the authors used to conjugate the NIRF dye sulfocyanine7 (Ex/Em, 750/773 $\mathrm{nm})$ and 2 targeting ligands. Both ${ }^{68} \mathrm{Ga}$-sulfo-Cy7-FSC-MG $\left(\mathrm{IC}_{50}, 2.68 \pm 0.53 \mathrm{nM} ; \log \mathrm{D},-1.9 \pm 0.17\right)$ and ${ }^{68} \mathrm{Ga}$-sulfoCy7-FSC-RGD ( $\left.\mathrm{IC}_{50}, 0.81 \pm 0.19 \mathrm{nM} ; \log \mathrm{D},-2.3 \pm 0.16\right)$ showed high receptor affinity and highly specific targeting properties in vitro. Both agents revealed in vivo accumulation in mouse xenografts as determined by PET/CT imaging and confirmed by biodistribution studies. ${ }^{68} \mathrm{Ga}$-sulfo-Cy7FSC-MG showed slower pharmacokinetics and higher liver accumulation $(25.7 \pm 3.3 \% \mathrm{ID} / \mathrm{g}$ vs. $6-7 \% \mathrm{ID} / \mathrm{g})$ than ${ }^{68} \mathrm{Ga}-$ sulfo-Cy7-FSC-RGD up to $2 \mathrm{~h}$. Optical imaging using ${ }^{68} \mathrm{Ga}-$ sulfo-Cy7-FSC-MG at delayed time points $(24-72 \mathrm{~h}$ ) improved in vivo tumor contrast, whereas ${ }^{68} \mathrm{Ga}$-sulfo-Cy7FSC-RGD had washed out from tumor tissue. The findings demonstrated the feasibility of using the FSC scaffold, as well as the need to improve the pharmacokinetic profile of FSC to support imaging at early time points. 


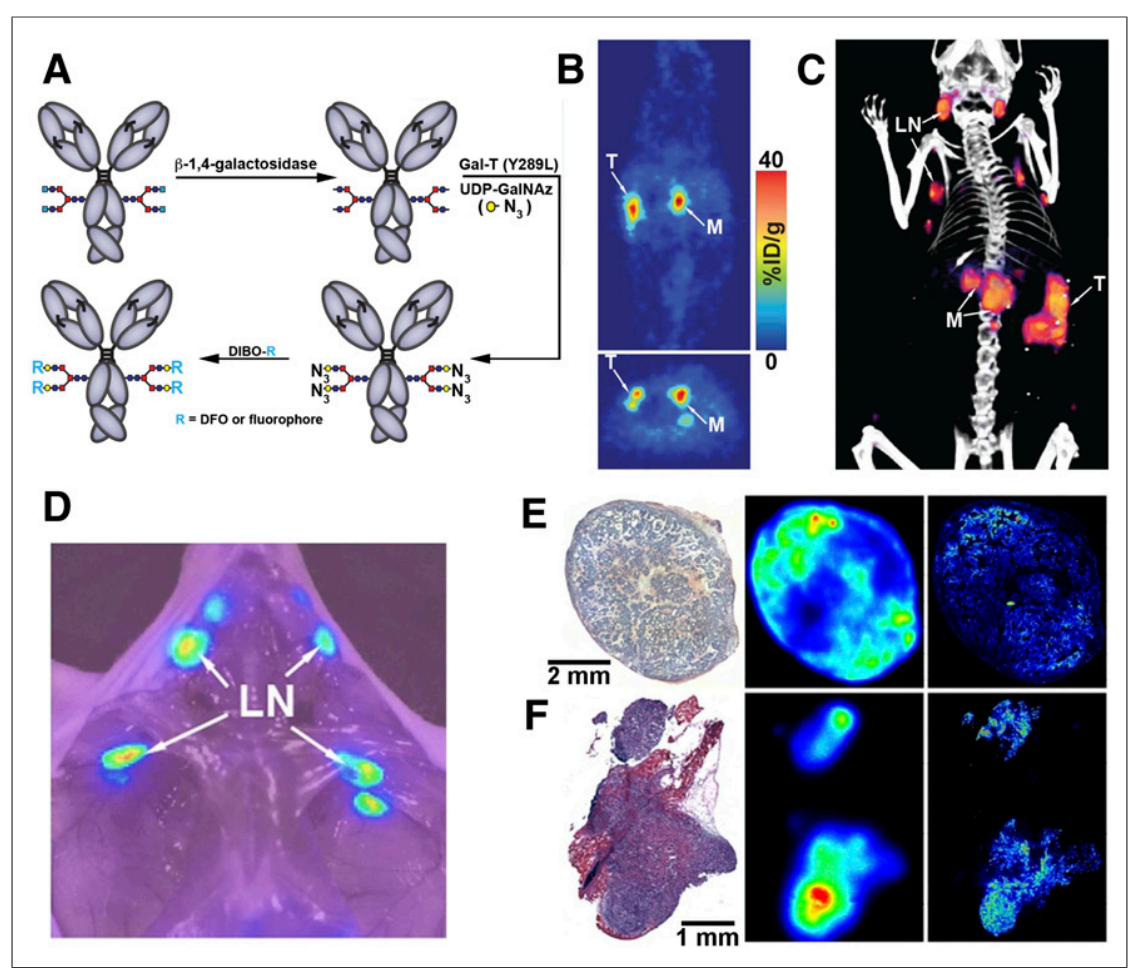

FIGURE 2. Site-specific conjugation and preclinical evaluation of ${ }^{89} \mathrm{Zr}$-ssDual-5B1. (A) Application of glycan engineering and bioorthogonal click-chemistry for synthesis of site-specific dual-labeled immunoconjugate. (B-D) PET, PET/CT, and NIRF imaging of orthotopic pancreatic ductal adenocarcinoma model at $120 \mathrm{~h}$ after injection demonstrate potential of ${ }^{89} \mathrm{Zr}$-ssDual-5B1 for identifying primary tumor (T), metastasis $(\mathrm{M})$, and sentinel lymph nodes (LN). (E and F) Histology (left), autoradiography (center), and fluorescence microscopy (right) of resected tissue from mice injected with ${ }^{89} \mathrm{Zr}$-ss Dual-5B1 confirm colocalization with cancer antigen 19-9 expression. (E) BxPC3 xenograft. (F) Metastatic foci. Adapted from (17).

Ghosh et al. also developed a modular dual labeling strategy but instead applied a translational focus that centered on using a clinically validated radiotracer as the model for a dual-labeled counterpart. The authors conjugated IRDye 800 to the somatostatin receptor subtype-2 (SSTR2)-targeted peptide DOTATOC using a multimodality chelation (MMC) scaffold that acted as a DOTA mimetic and enabled sitespecific conjugation of dye and peptide (Fig. 3A). Initially, $\mathrm{Cu} /{ }^{64} \mathrm{Cu}$-labeled MMC(IRDye800)-TOC was found to retain pharmacologic properties (cyclic adenosine monophosphate inhibition $\mathrm{EC}_{50}, 0.21 \pm 0.18 \mathrm{nM}$; receptor internalization $\left.\mathrm{EC}_{50}, 41.9 \pm 29.8 \mathrm{nM}\right)$ and in vitro receptor-targeting properties (21). PET/CT imaging showed SSTR2 uptake of ${ }^{64} \mathrm{Cu}-$ MMC(IRDye800)-TOC (Fig. 3B) that could be blocked in the presence of unlabeled octreotide, indicating receptor-mediated binding. The authors then synthesized a second-generation agent using an MMC analog with a modified pendant arm that contained a carboxyl group for stable chelation of ${ }^{68} \mathrm{Ga}$ (22). In vitro studies confirmed retention of SSTR2binding properties as shown by the ability of the agent to maximally inhibit cyclic adenosine monophosphate formation $\left(\mathrm{EC}_{50}, 0.066 \pm 0.012 \mathrm{nM}\right)$ and stimulate receptor internalization $\left(\mathrm{EC}_{50}, 48.7 \pm 9.9 \mathrm{nM}\right)$, as well as receptor-mediated uptake that was demonstrated by confocal microscopy and quantitative uptake assays. Preliminary in vivo assessment in healthy mice showed slower clearance from blood and higher uptake in normal organs than that of the parent compound, ${ }^{68} \mathrm{Ga}$ DOTATOC, suggesting the need for imaging at later times.

Baranski et al. also used a clinical radiotracer as a model system and reported the synthesis and evaluation of a library of dual-labeled agents derived from the clinically established radiotracer ${ }^{68} \mathrm{Ga}$-PSMA-11 for prostate-specific membrane antigen (PSMA) targeting (23). FITC (Ex/Em, 490/525 $\mathrm{nm})$, AlexaFluor488 (Ex/Em, 488/519 $\mathrm{nm}$ ), IRDye800, and DyLight800 (Ex/ Em, 769/795 nm) were conjugated to Glu-urea-Lys[Fe(HBED-CC)]-PEG $2^{-}$

$\mathrm{NH}_{2}$ and showed a cell uptake average ranging from $6 \%$ to $32 \%$ of total radioactivity added, with $\mathrm{IC}_{50}$ values ranging from $10.52 \pm 1.47 \mathrm{nM}$ to $35.54 \pm 2.94$ nM. PET/CT imaging of xenografted mice showed clear tumor accumulation using all 4 fluorescent-dye conjugates at $2 \mathrm{~h}$ after injection. Ex vivo biodistribution studies were in agreement and revealed prominent spleen accumulation. Because of the translational relevance of IRDye800, the authors further conducted a proof-of-concept for assessment of noncomplexed Glu-urea-Lys-HBED-CC-IRDye800 distribution in healthy pigs using the NIRF imaging capability of the robotic da Vinci surgical system (Intuitive Surgical, Inc.). Agent accumulation was observed only in PSMA-expressing organs, and results were confirmed ex vivo. This work demonstrated the feasibility of developing dual-labeled ${ }^{68} \mathrm{Ga}-$ PSMA-11 analogs that can extend the utility of preoperative PSMA PET scans into the operating room for improved surgical care in prostate cancer.

The performance of peptide-based dual-labeled analogs critically depends on the fluorescent label of choice and conjugation strategy. Recent reports have investigated how systematic modifications of the chemical composition of fluorescent dyes may dictate the in vivo characteristics of dual-labeled tracers. Bunschoten et al. developed a matrixbased scoring approach for evaluating the correlation among overall tracer charge, lipophilicity, and in vivo distribution (24). The authors synthesized a range of Cy5 (Ex/Em, 646/ $662 \mathrm{~nm}$ )-labeled hybrid c[RGDyK]-peptides, where systematic alkyl- and aromatic-substitutions of the dye were the only variable. They found that a moderate negative hydrophilicity in conjunction with a balanced charge distribution 


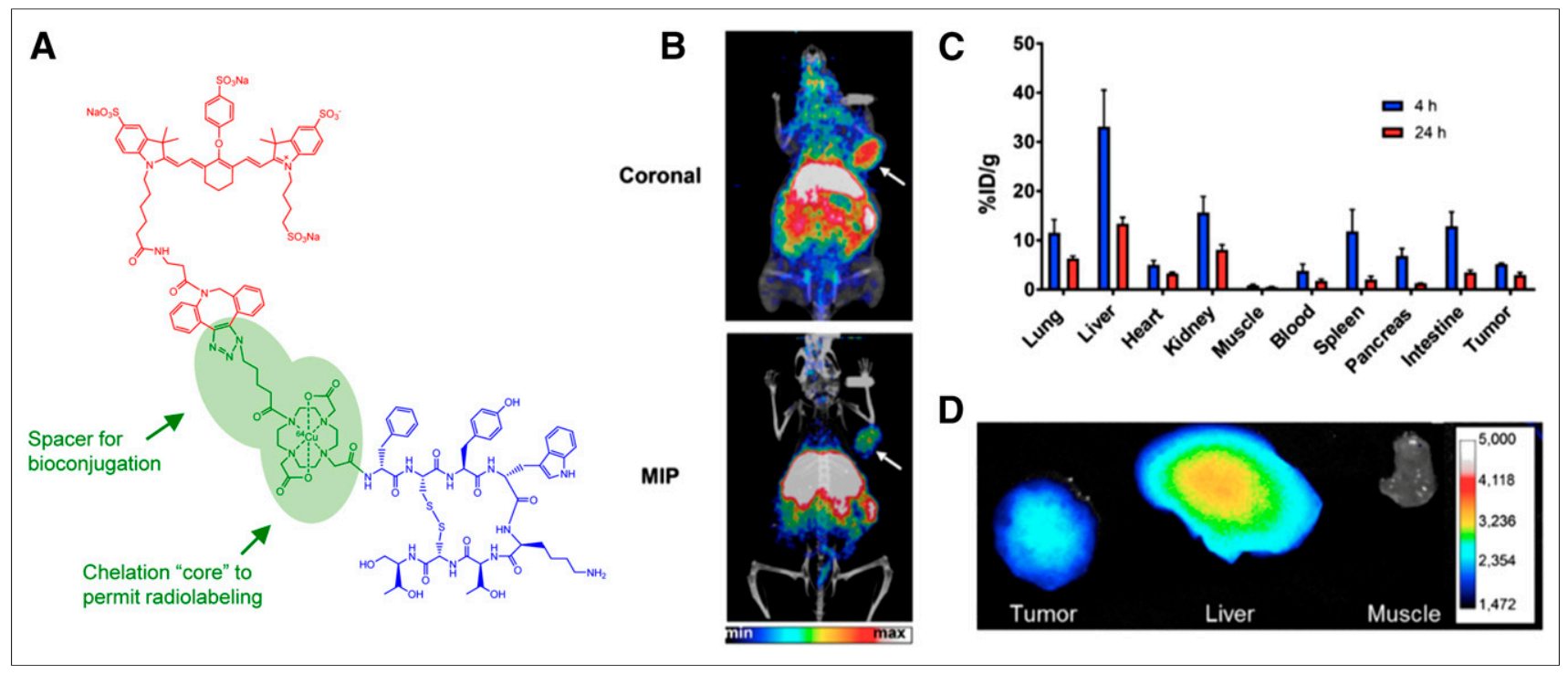

FIGURE 3. Structure and preclinical evaluation of SSTR2-targeted intraoperative imaging agent ${ }^{64} \mathrm{Cu}-\mathrm{MMC}(\mathrm{IRDye} 800)-\mathrm{TOC}$. (A) Components of ${ }^{64} \mathrm{Cu}-\mathrm{MMC}(\mathrm{IRDye800})-\mathrm{TOC}$. (B) PET and PET/CT imaging of representative AR24J xenograft at $24 \mathrm{~h}$ after injection. $(C$ and $D)$ Ex vivo analysis of tissues by quantification of radioactivity with y-counter (C) and NIRF imaging (D). Adapted from (21).

leads to improved pharmacokinetics. Importantly, it was shown that the systematic assessment of chemical modifications of the dye might allow the tailoring of tracers for improved targeting and faster clearance.

In another study, Buckle et al. elucidated how the elongation of the polymethine chain of cyanine dyes alters the pharmacokinetic disposition of otherwise similar dual-labeled tracers (25). Cy3 (Ex/Em, 550/570 nm), Cy5, and Cy7 were conjugated to DTPA-labeled c[RGDyK], and their bioactivity was confirmed in vitro. Significant differences in quantum yield and in vivo agent distributions were observed, which the authors partly attributed to differences in protein binding. Subsequent ex vivo biodistribution studies revealed comparable distribution profiles for the $\mathrm{Cy} 3$ - and Cy5-labeled counterparts, whereas ${ }^{111}$ In-DTPA-Cy7-c[RGDyK] showed improved tumor accumulation along with higher kidney and liver clearance. In a direct comparison, the intermediate-length tracer ${ }^{111}$ In-DTPA-Cy5-c[RGDyK] was found to provide the highest tumor-to-background ratios. These results emphasize the need for careful assessment of dye selection while understanding the impact on in vivo behavior.

\section{DUAL-LABELED AGENTS IN THE CLINIC}

Extensive preclinical evaluation of the carbonic IX-targeting antibody ${ }^{111}$ In-DOTA-girentuximab-IRDye800 (2628 ) has led to the initiation of a phase I clinical study to assess the feasibility and safety of intraoperative multimodal guidance in clear cell renal cell carcinoma patients (29). Dose escalation $(5,10,30$, and $50 \mathrm{mg}$ of antibody; each possessing $100 \mathrm{MBq}$ of radioactivity) was performed on patients scheduled for surgery, and SPECT/CT imaging at $4 \mathrm{~d}$ was followed by intraoperative guidance with a $\gamma$-probe and NIRF camera $7 \mathrm{~d}$ after injection. The agent was well tolerated at all doses, and excellent concordance was observed between SPECT/CT and NIRF imaging (Fig. 4). A mean tumor-to-normal-kidney ratio of $2.5 \pm 0.8$ was determined in tumors that expressed carbonic IX, compared with $1.0 \pm 0.1$ in tumors that lacked target expression, indicating specific, receptor-mediated uptake that provided clear fluorescent contrast at tumor margins. Although no significant differences were found in the amount of protein injected $(P=0.22)$, a trend toward a lower tumor-to-normal-kidney ratio was observed with higher doses. This trend could be explained by the fact that fluorescent agents

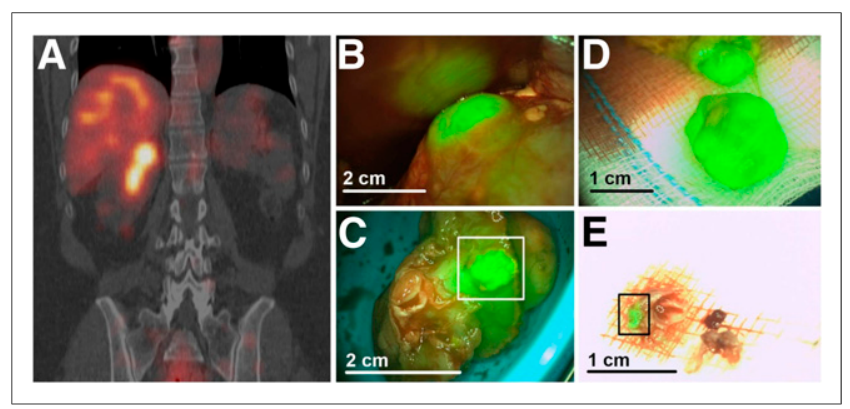

FIGURE 4. First-in-human dual-modality imaging of $111 \mathrm{In}$ DOTA-girentuximab-IRDye800. (A) Preoperative SPECT/CT for surgical planning of carbonic IX-overexpressing lesions $4 \mathrm{~d}$ after administration of $50 \mathrm{mg}$ of radiolabeled immunoconjugate (100 MBq). (B) Intraoperative NIRF imaging of tumors. (C) NIRF imaging of resected tumor containing surgical margins (box), subsequently confirmed by histopathology. (D) NIRF demonstration that further resection contained vital tumor, again confirmed by histopathology. (E) Evaluation of additionally removed tumor fragments by NIRF imaging (box). Histopathology confirmed that fragment consisted mainly of fibrotic tissue but also 2-mm tumor fragment. Adapted from (29). 
consist of a single chemical entity, unlike a radiotracer formulation that comprises the radiolabeled product and an unlabeled precursor. Therefore, administering higher protein (or precursor) amounts of a dual-labeled agent could lead to saturation of binding sites and intrinsic competition that may reduce the radioactive signal. Importantly, this study identified an optimal protein dose of $10 \mathrm{mg}$ and successfully demonstrated the safety and feasibility of ${ }^{111}$ In-DOTA-girentuximab-IRDye800 for intraoperative imaging of clear cell renal cell carcinoma. Moreover, the authors demonstrated techniques for the complementary use of the radioactive signal for noninvasive surgical planning and localization of occult clear cell renal cell carcinoma lesions intraoperatively with a $\gamma$-probe and the fluorescent signal for realtime visualization.

In another first-in-human study, $\mathrm{Li}$ et al. reported the synthesis and application of the dual-labeled peptide ${ }^{68} \mathrm{Ga}$ IRDye800-BBN for preoperative PET and intraoperative NIRF targeting of gastrin-releasing peptide receptor in glioblastoma multiforme (30). The feasibility of the multimodal agent was demonstrated in preclinical studies and then translated into patients to examine its ability to differentiate glioblastoma multiforme from normal brain tissue during surgery. A $40-\mu \mathrm{g}$ dose of ${ }^{68} \mathrm{Ga}$-IRDye800-BBN (74-148 $\mathrm{MBq}$ ) was used for preoperative PET studies, and $1 \mathrm{mg}$ of nonradioactive IRDye800-BBN was administered $2 \mathrm{~h}$ before the surgical procedure. The clinical data showed an excellent correlation between pre- and intraoperative signals. In vivo signal-to-background ratios were greater than 3 and did not differ from ex vivo signal-to-background ratios $(P>0.05)$. Sensitivity and specificity of $93.9 \%(95 \%$ confidence interval, $79.8 \%-99.3 \%$ ) and $100 \%$ (95\% confidence interval, 66.4\%-100\%), respectively, were reported. The agent was found to be safe, without development of additional neurologic deficits. Importantly, the combination of agent localization in tumors and penetration of NIRF signal allowed surgeons to identify residual lesions that were several millimeters beneath the tissue surface and increased the likelihood of achieving complete resections.

\section{PERSPECTIVE}

Intraoperative imaging is a rich area of research that is focused on improving the surgical standard of care for cancer. Advances in intraoperative imaging devices have played a critical role in the translation of FGS approaches by permitting highly sensitive detection of fluorescent agents administered at microdoses (i.e., subpharmacologic), thus minimizing toxicity concerns that might be present at higher dose levels. Although numerous nanoparticle-based approaches for dual labeling have been described, translational agent development efforts have predominantly used antibodies and peptides to capitalize on targeting vectors that are biocompatible and have established applications in clinical medicine. Taken together, the field possesses a relatively well-defined roadmap for translating an agent from the bench to the clinic, allowing new areas of research that are centered on optimizing the in vivo performance of FGS agents. Multimodality agents are at the cutting edge of this effort because of their ability to combine the strengths of noninvasive preoperative imaging, radioguided surgery, intraoperative fluorescence imaging, and surgical pathology. Although the presence of the radiolabel has clear utility in patient studies, its major contribution could potentially emerge in preclinical studies that evaluate agent optimization strategies through head-to-head comparison of full-length mAbs versus fragments, receptors agonists versus antagonists, linkers, and fluorescent dyes. The development of new dyes is indeed a critical area since it could conceivably allow an established FGS (or multimodality) agent to be upgraded by switching to a fluorophore with superior optical properties. This includes using dyes that either are charge-balanced or possess an enhanced charge distribution profile to reduce protein binding and nonspecific interactions with off-target sites. The zwitterionic dye ZW800 initially showed this effect (14) and is now accompanied by new classes of NIRF cyanine dyes that use a $\mathrm{C}^{\prime}-O$-alkyl linker and produced more favorable in vivo imaging characteristics than a netnegatively charged counterpart (31). Improving the penetration depth of the optical signal in current dual-labeled agents is another growing area of optics research, and a molecular platform that combines NIR-II $\left(\lambda_{\mathrm{em}}, 1,000-1,700 \mathrm{~nm}\right)$ and PET isotopes with high chemoselectivity was reported by Sun et al. (32). The authors conducted a proof-of-concept study in which RGD was conjugated to the triaza bifunctional chelator NOTA and a NIR-II dye (Ex/Em, 808/1055 $\mathrm{nm})$ to produce ${ }^{68} \mathrm{Ga}-\mathrm{CHS} 2$. Using NIR-II imaging, the highest tumor contrast was determined at $12 \mathrm{~h}$ after agent administration (tumor-to-normal-tissue ratio, $4.77 \pm 0.26$ ) and was 2-fold higher than previously reported NIRF/RGD agents. These studies provide examples of new reagents that might be used for dual labeling and show how chemical optimization strategies can improve tumor detection intraoperatively.

\section{DISCLOSURE}

This work was supported by the National Institute of Biomedical Imaging and Bioengineering (R01 EB017279) and an endowment from the John S. Dunn Foundation (both to Ali Azhdarinia). No other potential conflict of interest relevant to this article was reported.

\section{REFERENCES}

1. Orosco RK, Tapia VJ, Califano JA, et al. Positive surgical margins in the 10 most common solid cancers. Sci Rep. 2018;8:5686.

2. Wyld L, Audisio RA, Poston GJ. The evolution of cancer surgery and future perspectives. Nat Rev Clin Oncol. 2015;12:115-124.

3. van Dam GM, Themelis G, Crane LM, et al. Intraoperative tumor-specific fluorescence imaging in ovarian cancer by folate receptor-alpha targeting: first in-human results. Nat Med. 2011;17:1315-1319.

4. Pogue BW, Rosenthal EL, Achilefu S, van Dam GM. Perspective review of what is needed for molecular-specific fluorescence-guided surgery. J Biomed Opt. 2018;23: $1-9$. 
5. Azhdarinia A, Ghosh P, Ghosh S, Wilganowski N, Sevick-Muraca EM. Duallabeling strategies for nuclear and fluorescence molecular imaging: a review and analysis. Mol Imaging Biol. 2012;14:261-276.

6. Kuil J, Velders AH, van Leeuwen FW. Multimodal tumor-targeting peptides functionalized with both a radio- and a fluorescent label. Bioconjug Chem. 2010;21: 1709-1719.

7. Lütje S, Rijpkema M, Helfrich W, Oyen WJ, Boerman OC. Targeted radionuclide and fluorescence dual-modality imaging of cancer: preclinical advances and clinical translation. Mol Imaging Biol. 2014;16:747-755.

8. van der Poel HG, Buckle T, Brouwer OR, Valdes Olmos RA, van Leeuwen FW. Intraoperative laparoscopic fluorescence guidance to the sentinel lymph node in prostate cancer patients: clinical proof of concept of an integrated functional imaging approach using a multimodal tracer. Eur Urol. 2011;60:826-833.

9. KleinJan GH, van Werkhoven E, van den Berg NS, et al. The best of both worlds: a hybrid approach for optimal pre- and intraoperative identification of sentinel lymph nodes. Eur J Nucl Med Mol Imaging. 2018;45:1915-1925.

10. Hekman MCH, Rijpkema M, Bos DL, et al. Detection of micrometastases using SPECT/fluorescence dual-modality imaging in a CEA-expressing tumor model. J Nucl Med. 2017;58:706-710.

11. Odenthal J, Rijpkema M, Bos D, et al. Targeting CD44v6 for fluorescence-guided surgery in head and neck squamous cell carcinoma. Sci Rep. 2018;8:10467.

12. Luo H, Hernandez R, Hong $\mathrm{H}$, et al. Noninvasive brain cancer imaging with a bispecific antibody fragment, generated via click chemistry. Proc Natl Acad Sci USA. 2015;112:12806-12811.

13. Luo H, England CG, Goel S, et al. ImmunoPET and near-infrared fluorescence imaging of pancreatic cancer with a dual-labeled bispecific antibody fragment. Mol Pharm. 2017;14:1646-1655.

14. Choi HS, Nasr K, Alyabyev S, et al. Synthesis and in vivo fate of zwitterionic near-infrared fluorophores. Angew Chem Int Ed Engl. 2011;50:6258-6263.

15. Gao P, Pinkston KL, Wilganowski N, et al. Deglycosylation of mAb by EndoS for improved molecular imaging. Mol Imaging Biol. 2015;17:195-203.

16. Zeglis BM, Davis CB, Abdel-Atti D, et al. Chemoenzymatic strategy for the synthesis of site-specifically labeled immunoconjugates for multimodal PET and optical imaging. Bioconjug Chem. 2014;25:2123-2128.

17. Houghton JL, Zeglis BM, Abdel-Atti D, et al. Site-specifically labeled CA19.9targeted immunoconjugates for the PET, NIRF, and multimodal PET/NIRF imaging of pancreatic cancer. Proc Natl Acad Sci USA. 2015;112:15850-15855.

18. Lu Z, Pham TT, Rajkumar V, et al. A dual reporter iodinated labeling reagent for cancer positron emission tomography imaging and fluorescence-guided surgery. J Med Chem. 2018;61:1636-1645.
19. Zhang H, Desai P, Koike Y, et al. Dual-modality imaging of prostate cancer with a fluorescent and radiogallium-labeled gastrin-releasing peptide receptor antagonist. J Nucl Med. 2017;58:29-35.

20. Summer D, Grossrubatscher L, Petrik M, et al. Developing targeted hybrid imaging probes by chelator scaffolding. Bioconjug Chem. 2017;28:1722-1733.

21. Ghosh SC, Rodriguez M, Carmon KS, et al. A modular dual-labeling scaffold that retains agonistic properties for somatostatin receptor targeting. J Nucl Med. 2017;58:1858-1864.

22. Ghosh SC, Hernandez Vargas S, Rodriguez M, et al. Synthesis of a fluorescently labeled ${ }^{68} \mathrm{Ga}$-DOTA-TOC analog for somatostatin receptor targeting. ACS Med Chem Lett. 2017;8:720-725.

23. Baranski AC, Schafer M, Bauder-Wust U, et al. PSMA-11-derived dual-labeled PSMA inhibitors for preoperative PET imaging and precise fluorescence-guided surgery of prostate cancer. J Nucl Med. 2018;59:639-645.

24. Bunschoten A, van Willigen DM, Buckle T, et al. Tailoring fluorescent dyes to optimize a hybrid RGD-tracer. Bioconjug Chem. 2016;27:1253-1258.

25. Buckle T, van Willigen DM, Spa SJ, et al. Tracers for fluorescence-guided surgery: how elongation of the polymethine chain in cyanine dyes alters the pharmacokinetics of a dual-modality c[RGDyK] tracer. J Nucl Med. 2018;59:986-992.

26. Muselaers $\mathrm{CH}$, Stillebroer AB, Rijpkema M, et al. Optical imaging of renal cell carcinoma with anti-carbonic anhydrase IX monoclonal antibody girentuximab. J Nucl Med. 2014;55:1035-1040.

27. Muselaers $\mathrm{CH}$, Rijpkema M, Bos DL, et al. Radionuclide and fluorescence imaging of clear cell renal cell carcinoma using dual labeled anti-carbonic anhydrase IX antibody G250. J Urol. 2015;194:532-538.

28. Hekman MC, Boerman OC, de Weijert M, et al. Targeted dual-modality imaging in renal cell carcinoma: an ex vivo kidney perfusion study. Clin Cancer Res. 2016;22: $4634-4642$.

29. Hekman MC, Rijpkema M, Muselaers $\mathrm{CH}$, et al. Tumor-targeted dual-modality imaging to improve intraoperative visualization of clear cell renal cell carcinoma: a first in man study. Theranostics. 2018;8:2161-2170.

30. Li D, Zhang J, Chi C, et al. First-in-human study of PET and optical dualmodality image-guided surgery in glioblastoma using ${ }^{68} \mathrm{Ga}$-IRDye $800 \mathrm{CW}$ BBN. Theranostics. 2018;8:2508-2520.

31. Sato K, Gorka AP, Nagaya T, et al. Role of fluorophore charge on the in vivo optical imaging properties of near-infrared cyanine dye/monoclonal antibody conjugates. Bioconjug Chem. 2016;27:404-413.

32. Sun Y, Zeng X, Xiao Y, et al. Novel dual-function near-infrared II fluorescence and PET probe for tumor delineation and image-guided surgery. Chem Sci. 2018;9: 2092-2097. 